Supplement of Hydrol. Earth Syst. Sci., 19, 3033-3045, 2015

http://www.hydrol-earth-syst-sci.net/19/3033/2015/

doi:10.5194/hess-19-3033-2015-supplement

(C) Author(s) 2015. CC Attribution 3.0 License.

(c) (i)

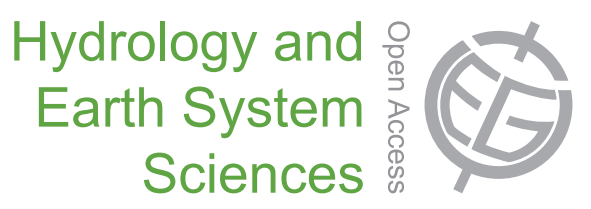

Supplement of

\title{
Time series analysis of the long-term hydrologic impacts of afforestation in the Águeda watershed of north-central Portugal
}

D. Hawtree et al.

Correspondence to: D. Hawtree (daniel.hawtree@mailbox.tu-dresden.de)

The copyright of individual parts of the supplement might differ from the CC-BY 3.0 licence. 

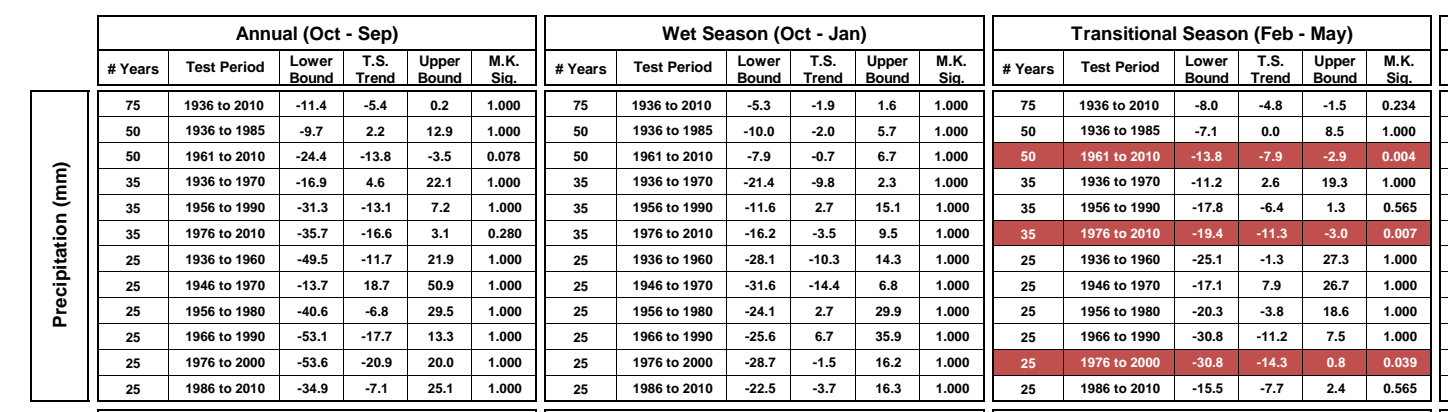

\begin{tabular}{|c|c|c|c|c|c|}
\hline \multicolumn{7}{|c|}{ Dry Season (Jun - Sep) } \\
\hline Years & Test Period & $\begin{array}{l}\text { Lower } \\
\text { Bound }\end{array}$ & $\begin{array}{c}\text { T.S. } \\
\text { Trend }\end{array}$ & $\begin{array}{c}\text { Upper } \\
\text { Bound }\end{array}$ & $\begin{array}{c}\text { M.K. } \\
\text { Sia. }\end{array}$ \\
\hline 75 & 1936 to 2010 & -1.1 & 0.0 & 0.9 & 1.000 \\
\hline 50 & 1936 to 1985 & -1.7 & 0.3 & 2.1 & 1.000 \\
\hline 50 & 1961 to 2010 & -2.7 & -0.8 & 1.2 & 1.000 \\
\hline 35 & 1936 to 1970 & -2.0 & 1.1 & 3.8 & 1.000 \\
\hline 35 & 1956 to 1990 & -6.3 & -2.4 & 1.1 & 1.000 \\
\hline 35 & 1976 to 2010 & -3.1 & 0.5 & 3.8 & 1.000 \\
\hline 25 & 1936 to 1960 & -4.5 & 1.4 & 6.8 & 1.000 \\
\hline 25 & 1946 to 1970 & -2.2 & 2.6 & 7.2 & 1.000 \\
\hline 25 & 1956 to 1980 & -10.2 & -5.2 & 1.1 & 1.000 \\
\hline 25 & 1966 to 1990 & -6.7 & -1.9 & 2.9 & 1.000 \\
\hline 25 & 1976 to 2000 & -4.1 & 1.2 & 7.2 & 1.000 \\
\hline 25 & 1986 to 2010 & -6.9 & -1.5 & 4.2 & 1.000 \\
\hline
\end{tabular}

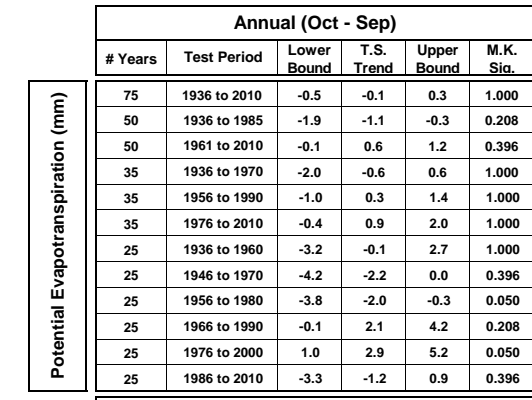

Wet Season (Oct - Jan)
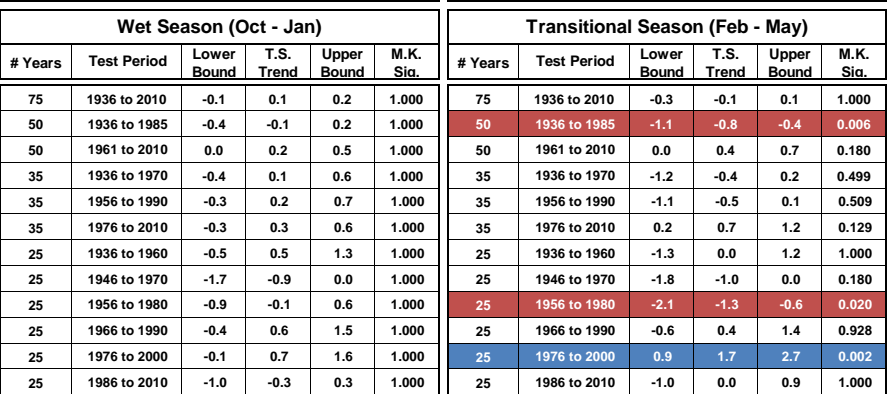

Wet Season (Oct - Jan)

\begin{tabular}{|c|c|c|c|c|c|}
\hline \multicolumn{5}{|c|}{ Annual* (Oct - Jul) } \\
\hline \# Years & Test Period & $\begin{array}{c}\text { Lower } \\
\text { Bound }\end{array}$ & $\begin{array}{c}\text { T.S. } \\
\text { Trend }\end{array}$ & $\begin{array}{c}\text { Upper } \\
\text { Bound }\end{array}$ & $\begin{array}{c}\text { M.K.K. } \\
\text { Siq. }\end{array}$ \\
\hline & & & &
\end{tabular}

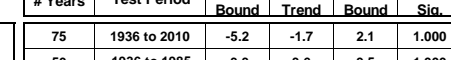

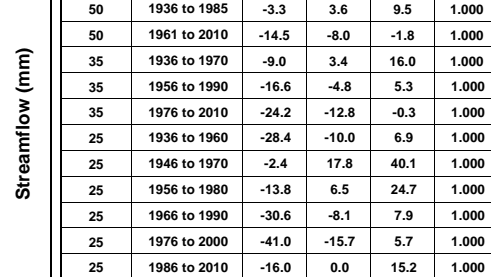
Annual $^{*}$ (Oct - May)

\begin{tabular}{|c|c|c|c|c|c|}
\hline \multicolumn{7}{|c|}{ Wet Season (Oct - Jan) } \\
\hline \# Years & Test Period & $\begin{array}{c}\text { Lower } \\
\text { Bound }\end{array}$ & $\begin{array}{c}\text { T.S. } \\
\text { Trend }\end{array}$ & $\begin{array}{c}\text { Upper } \\
\text { Bound }\end{array}$ & $\begin{array}{c}\text { M.K. } \\
\text { Siq. }\end{array}$ \\
\hline \hline 75 & 1936 to 2010 & -2.1 & -0.1 & 2.0 & 1.000 \\
\hline 50 & 1936 to 1985 & -2.8 & 0.9 & 4.7 & 1.000 \\
\hline 50 & 1961 to 2010 & -7.4 & -2.4 & 1.4 & 1.000 \\
\hline 35 & 1936 to 1970 & -7.6 & 0.3 & 8.1 & 1.000 \\
\hline 35 & 1956 to 1990 & -9.6 & -1.8 & 5.2 & 1.000 \\
\hline 35 & 1976 to 2010 & -11.1 & -2.2 & 3.6 & 1.000 \\
\hline 25 & 1936 to 1960 & -18.0 & -9.4 & -0.5 & 1.000 \\
\hline 25 & 1946 to 1970 & -2.0 & 10.6 & 22.2 & 1.000 \\
\hline 25 & 1956 to 1980 & -15.1 & -0.3 & 12.1 & 1.000 \\
\hline 25 & 1966 to 1990 & -17.8 & -4.0 & 6.5 & 1.000 \\
\hline 25 & 1976 to 2000 & -14.8 & -1.1 & 12.2 & 1.000 \\
\hline 25 & 1986 to 2010 & -11.1 & 1.6 & 14.1 & 1.000 \\
\hline
\end{tabular}

\begin{tabular}{|c|c|c|c|c|c|}
\hline \# Years & Test Period & $\begin{array}{c}\text { Lower } \\
\text { Bound }\end{array}$ & $\begin{array}{c}\text { T.S. } \\
\text { Trend }\end{array}$ & $\begin{array}{c}\text { Upper } \\
\text { Bound }\end{array}$ & $\begin{array}{c}\text { M.K. } \\
\text { Siq. }\end{array}$ \\
\hline
\end{tabular}

$$
\begin{array}{|l|c|c|c|c|c|}
\hline \hline \multicolumn{5}{|c|}{\text { Wet Season (Oct - Jan) }} \\
\hline \text { \# Years } & \text { Test Period } & \begin{array}{c}
\text { Lower } \\
\text { Bound }
\end{array} & \begin{array}{c}
\text { T.S. } \\
\text { Trend }
\end{array} & \begin{array}{l}
\text { Upper } \\
\text { Bound }
\end{array} & \begin{array}{c}
\text { M.K. } \\
\text { Siq. }
\end{array} \\
\hline \hline & & & & & \\
\hline
\end{array}
$$

\begin{tabular}{|c|c|c|c|c|c|}
\hline 25 & 1986 to 2010 & -1.0 & 0.0 & 0.9 & 1.000 \\
\hline \hline \multicolumn{5}{c|}{ Transitional Season (Feb - May) } \\
\hline
\end{tabular}

\begin{tabular}{|l|c|c|c|c|c|}
\hline \# Years & Test Period & Lower & T.S. & Upper & M.K. \\
\hline
\end{tabular}

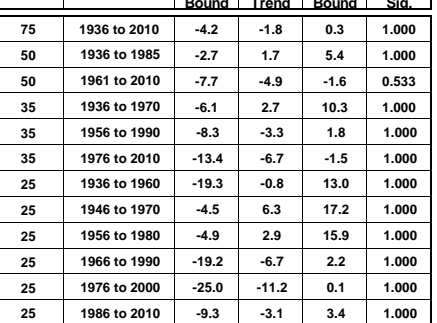

\begin{tabular}{|c|c|c|c|c|c|}
\hline \hline \multicolumn{5}{|c|}{ Transitional Season (Feb - May) } \\
\hline \# Years & Test Period & Lower & $\begin{array}{c}\text { T.S. } \\
\text { Bound } \\
\text { Trend }\end{array}$ & $\begin{array}{l}\text { Upper } \\
\text { Bound }\end{array}$ & $\begin{array}{c}\text { M.K. } \\
\text { Siq. }\end{array}$ \\
\hline
\end{tabular}

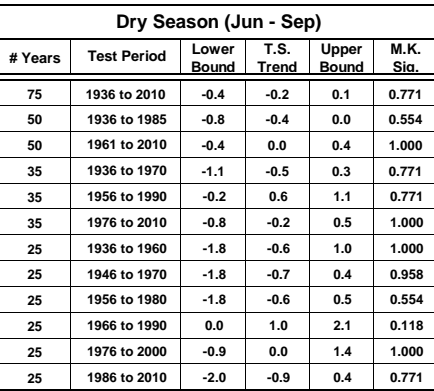

\begin{tabular}{|c|c|c|c|c|c|}
\hline 25 & 1976 to 2000 & -0.9 & 0.0 & 1.4 & 1.000 \\
\hline 25 & 1986 to 2010 & -2.0 & -0.9 & 0.4 & 0.771 \\
\hline
\end{tabular}

\section{Dry Season (Jun - Sep)}

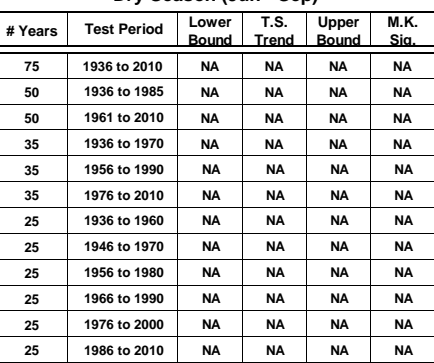

Dry Season (Jun - Sep)

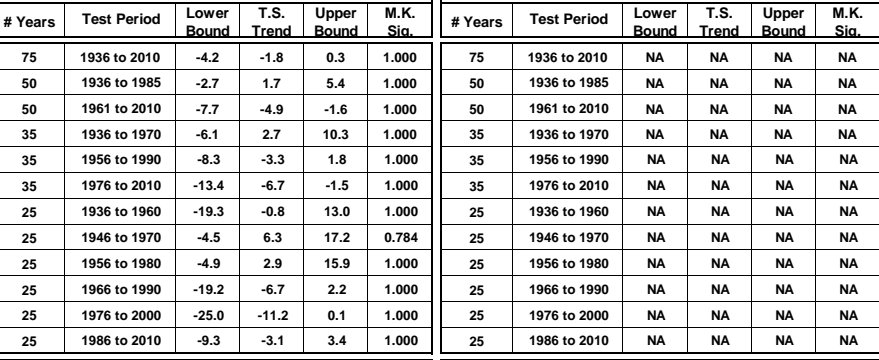

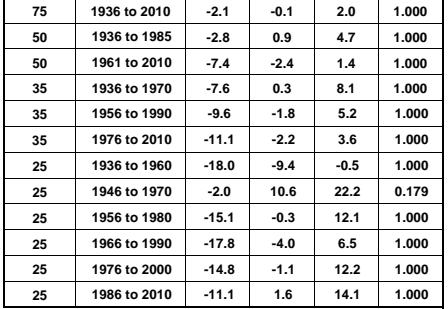

$$
\text { Annual* (Oct - May) }
$$

\begin{tabular}{|l|c|c|c|c|c|}
\hline \# Years & Test Period & $\begin{array}{l}\text { Lower } \\
\text { Bound }\end{array}$ & $\begin{array}{c}\text { T.S. } \\
\text { Trend }\end{array}$ & $\begin{array}{c}\text { Upper } \\
\text { Bound }\end{array}$ & $\begin{array}{c}\text { M.K. } \\
\text { Sia. }\end{array}$ \\
\hline
\end{tabular}

$$
\begin{array}{|l|l|l|l|l|l|}
\hline 25 & 1986 \text { to } 2010 & -11.1 & 1.6 & 14.1 & 1.000 \\
\hline \multicolumn{5}{|c|}{\text { Wet Season (Oct - Jan) }} \\
\hline
\end{array}
$$

Transitional Season (Feb - May)

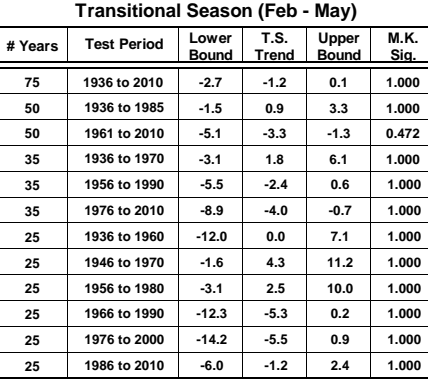

Wet Season (Oct - Jan)

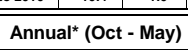

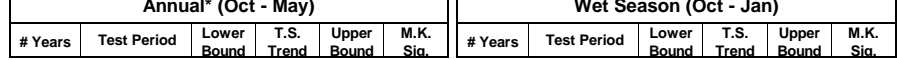

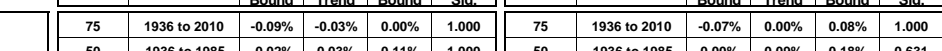

\begin{tabular}{|c|c|c|c|c|c|}
\hline 50 & 1936 to 1985 & $-0.02 \%$ & $0.03 \%$ & $0.11 \%$ & 1.000 \\
\hline 50 & 1961 to 2010 & $-0.33 \%$ & $-0.20 \%$ & $-0.09 \%$ & 0.792 \\
\hline & & & & \\
\hline
\end{tabular}

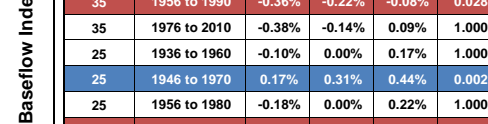

\begin{tabular}{|c|c|c|c|c|c|c|c|c|c|c|c|}
\hline \multicolumn{6}{|c|}{ Transitional Season (Feb - May) } & \multicolumn{6}{|c|}{ Dry Season (Jun - Sep) } \\
\hline \# Years & Test Period & $\begin{array}{l}\text { Lower } \\
\text { Bound }\end{array}$ & $\begin{array}{l}\text { T.S. } \\
\text { Trend }\end{array}$ & $\begin{array}{l}\text { Upper } \\
\text { Bound }\end{array}$ & $\begin{array}{l}\text { M.K. } \\
\text { Sia. }\end{array}$ & \# Years & Test Period & $\begin{array}{l}\text { Lower } \\
\text { Bound }\end{array}$ & $\begin{array}{l}\text { T.S. } \\
\text { Trend }\end{array}$ & $\begin{array}{l}\text { Upper } \\
\text { Bound }\end{array}$ & $\begin{array}{l}\text { M.K. } \\
\text { Sia. }\end{array}$ \\
\hline 75 & 1936 to 2010 & $-0.20 \%$ & $-0.09 \%$ & $0.00 \%$ & 0.743 & 75 & 1936 to 2010 & $\mathrm{NA}$ & NA & $\mathrm{NA}$ & NA \\
\hline 50 & 1936 to 1985 & $-0.23 \%$ & $-0.07 \%$ & $0.07 \%$ & 1.000 & 50 & 1936 to 1985 & $\mathrm{NA}$ & $\mathrm{NA}$ & $\mathrm{NA}$ & $\mathrm{NA}$ \\
\hline 50 & 1961 to 2010 & $-0.35 \%$ & $-0.16 \%$ & $0.00 \%$ & 1.000 & 50 & 1961 to 2010 & NA & NA & NA & NA \\
\hline 35 & 1936 to 1970 & $-0.36 \%$ & $-0.04 \%$ & $0.19 \%$ & 1.000 & 35 & 1936 to 1970 & $\mathrm{NA}$ & $\mathrm{NA}$ & $\mathrm{NA}$ & $\mathrm{NA}$ \\
\hline 35 & 1956 to 1990 & $-0.36 \%$ & $-0.14 \%$ & $0.05 \%$ & 0.743 & 35 & 1956 to 1990 & $\mathrm{NA}$ & $\mathrm{NA}$ & $\mathrm{NA}$ & $\mathrm{NA}$ \\
\hline 35 & 1976 to 2010 & $-0.40 \%$ & $0.00 \%$ & $0.29 \%$ & 1.000 & 35 & 1976 to 2010 & NA & NA & NA & NA \\
\hline 25 & 1936 to 1960 & $-0.75 \%$ & $-0.26 \%$ & $0.18 \%$ & 1.000 & 25 & 1936 to 1960 & NA & NA & NA & NA \\
\hline 25 & 1946 to 1970 & $-0.11 \%$ & $0.20 \%$ & $0.58 \%$ & 1.000 & 25 & 1946 to 1970 & NA & NA & $\mathrm{NA}$ & NA \\
\hline 25 & 1956 to 1980 & $-0.25 \%$ & $0.12 \%$ & $0.40 \%$ & 1.000 & 25 & 1956 to 1980 & NA & NA & $\mathrm{NA}$ & NA \\
\hline 25 & 1966 to 1990 & $-0.88 \%$ & $-0.35 \%$ & $0.05 \%$ & 0.743 & 25 & 1966 to 1990 & $\mathrm{NA}$ & $\mathrm{NA}$ & $\mathrm{NA}$ & $\mathrm{NA}$ \\
\hline 25 & 1976 to 2000 & $-0.06 \%$ & $0.29 \%$ & $0.71 \%$ & 0.743 & 25 & 1976 to 2000 & $\mathrm{NA}$ & $\mathrm{NA}$ & $\mathrm{NA}$ & $\mathrm{NA}$ \\
\hline 25 & 1986 to 2010 & $-0.53 \%$ & $0.00 \%$ & $0.73 \%$ & 1.000 & 25 & 1986 to 2010 & $\mathrm{NA}$ & $\mathrm{NA}$ & $\mathrm{NA}$ & $\mathrm{NA}$ \\
\hline
\end{tabular}

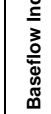

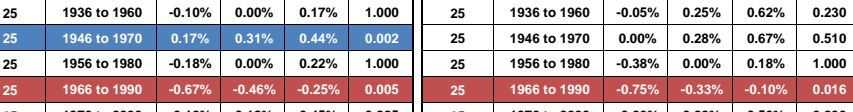
\begin{tabular}{|l|l|l|l|l|l|l|l|l|l|l|l|l|}
\hline 25 & 1976 to 2000 & $-0.12 \%$ & $-0.48 \%$ & $-0.25 \%$ & 0.005 & 25 & 1966 to 1990 & $-0.75 \%$ & $-0.33 \%$ & $-0.10 \%$ & 0.016 \\
\hline 25 & 0.885 & 2576 to 2000 & $-0.20 \%$ & $0.22 \%$ & $0.50 \%$ & 0.230 \\
\hline
\end{tabular} \begin{tabular}{|c|c|c|c|c|c|c|c|c|c|c|}
\hline 25 & 1976 to 2000 & $-0.12 \%$ & $0.18 \%$ & $0.45 \%$ & 0.885 \\
\hline 25 & 19010 & $-0.50 \%$ & $-0.11 \%$ & $0.42 \%$ & 1.000 \\
\hline 25 & 1976 to 2000 & $-0.20 \%$ & $0.22 \%$ & $0.50 \%$ & 0.230 \\
\hline 25 & 1986 to 2010 & $-0.62 \%$ & $-0.12 \%$ & $0.50 \%$ & 1.000 \\
\hline
\end{tabular}

Transitional Season (Feb - May) 